\title{
IMPRESSORA 3D NA FABRICAÇÃO DE MICROCANAIS E MICROMISTURADORES
}

\author{
A. C. RODRIGUES ${ }^{1}$, H. S. SANTANA ${ }^{1}$, O. P. TARANTO ${ }^{1}$ \\ ${ }^{1}$ Universidade Estadual de Campinas, Faculdade de Engenharia Química \\ E-mail para contato: alancr2014@gmail.com
}

\begin{abstract}
RESUMO - Este trabalho introduz o uso de impressora 3D para a produção de dispositivos com microcanais. Os limites da tecnologia de impressão 3D, que engloba a resolução de impressão, replicação e diferenças entre programas de processamento de modelos 3D foram estudados e caracterizados, obtendo fidelidade de replicação de impressão a partir de $200 \mu \mathrm{m}$ e limites mínimos aceitáveis para futuros trabalhos com microdispositivos e microcanais.
\end{abstract}

\section{INTRODUÇÃO}

Nos últimos anos foram realizados diversos estudos sobre reação química em microdispositivos explorando a influência do tipo de geometria, os efeitos da variação da temperatura, concentração de catalisador e razão molar, da relação entre o grau de mistura dos reagentes e eficiência reacional e o escalonamento de microunidades (i.e., multiplicação das microunidades desenvolvidas em laboratórios para obtenção de uma escala industrial) (Dai et al., 2014). Os microdispositivos normalmente são fabricados pelo processo de litografia macia. Apesar da técnica de litografia macia proporcionar a fabricação de estruturas com excelente definição dos padrões de microcanais, conforme relatou Coltro et al. (2007) o tempo dispendido para a produção de cada dispositivo e o custo de implementação de um laboratório e manutenção são altos, tornando inviável a fabricação de muitas unidades de microdispositivos para a produção comercial de produtos. Para tentar otimizar a produção de microdispositivos, ultimamente tem sido proposto o uso de impressoras 3D para a fabricação desses dispositivos, uma vez que a impressão 3D oferece a vantagem da cadeia de processo simplificada e a possibilidade de fabricar sistemas complexos de vários níveis (Lee et al., 2016).

As impressoras 3D são particularmente atraentes no campo das ciências químicas, uma vez que o seu método de fabricação permite um controle preciso sobre a topologia, geometria e composição do reator resultante sendo que a facilidade de uso, versatilidade e economia da nova geração de impressoras 3D são parte primordial deste processo (Kitson et al., 2016). A impressão 3D é uma técnica que fabrica objetos físicos a partir de modelos digitais por processos aditivos, através dos quais são depositadas sucessivas camadas de material para produzir o objeto final (Kitson et al., 2016). Diversos são os materiais que podem ser utilizados na impressão 3D, dentre eles, os polímeros mais utilizados neste método de impressão 3D são o poliácido lático (PLA) e o acrilonitrilo-butadieno-estireno (ABS) (Kitson et al., 2016). O PLA apresenta qualidades que permitem um bom estudo dos microdispositivos com reações químicas, pois mantem a sua integridade estrutural a $200{ }^{\circ} \mathrm{C} \mathrm{e}$ é quimicamente resistente em meios ácidos. Comparativamente, o PDMS, polímero utilizado para a produção de microreatores por litografia macia, é mais caro do que o PLA e apresenta 
algumas desvantagens, tais como baixo módulo de elasticidade, o que pode causar deformação geométrica em estruturas microfluídicas sob operação de alta pressão.

Uma das áreas que ainda a impressora 3D não foi explorada foi a fabricação de canais com misturadores ou micromisturadores, que elevam a eficiência reacional, além de permitir o aumento do tamanho do microdispositivo sem a perda de suas propriedades inerentes a escala micro. Diante disso, esse trabalho tem como objetivo demonstrar a aplicação de impressoras 3D na fabricação de microcanais e micromisturadores com a utilização de PLA.

\section{MATERIAIS E MÉTODOS}

As peças foram impressas através da impressora 3D (Sethi3D S3, Sethi3D, Campinas, SP, Brasil). A Sethi3D S3, faz uso de filamentos de $1,75 \mathrm{~mm}$, bico com saída de 0,4 $\mathrm{mm}$, com resolução ajustável de $50 \mu \mathrm{m}$ à $300 \mu \mathrm{m}$ de altura de camada. O volume limite de impressão é $270 \times 270 \times 320 \mathrm{~mm}$ para essa impressora. A tecnologia de impressão utilizada é a Fused Filament Fabrication (FFF), em que a impressora 3D utiliza-se de um filamento contínuo de um material termoplástico. O material é fundido e, por meio de uma bobina, é extrusado, saindo pelo bico do extrusor e depositado na mesa aquecedora ou na peça de trabalho. O bico é movido, sob os comandos contidos no arquivo de impressão, no plano horizontal e vertical para definir a forma impressa.

Os modelos digitais foram desenhados através do software Autodesk Inventor (Autodesk Inventor, Autodesk, Inc., San Rafael, CA, USA), que gerou os arquivos na extensão .stl. Esses arquivos em stl são utilizados em programas fatiadores que geram arquivos na extensão G-code, extensão reconhecida pelas impressoras 3D. Nesse trabalho foram verificados os limites de impressão da Sethi3D S3 que definem o tamanho mínimo que um microcanal pode ser impresso com fidelidade e qual são os valores mínimos e máximos para os obstáculos/micromisturadores. As caracterizações realizadas são descritas abaixo.

Foram realizados testes de comparação entre programas de processamento de modelos 3D em instruções de impressão 3D baseadas em GCode. Foram utilizados 3 programas para transformar os arquivos stl gerado pelo Autodesk Inventor em arquivos G-code: Simplify $3 \mathrm{D} \AA$, CuraEngine $\AA$ e Slic $3 \mathrm{r} \circledast$. As comparações entre os programas foram realizadas utilizando o processo de impressão 3D para fabricar dispositivos de caracterização, estruturas com $30 \times 30 \mathrm{~mm}$ de base, com um canal a $400 \mu \mathrm{m}$ da base, variando a profundidade do canal $(200 \mu \mathrm{m}, 400 \mu \mathrm{m}, 1000 \mu \mathrm{m}$ e $2000 \mu \mathrm{m})$ e as espessuras de camadas $(50 \mu \mathrm{m}, 100 \mu \mathrm{m}, 200 \mu \mathrm{m}$, $300 \mu \mathrm{m})$. Os dispositivos foram impressos para testar a resolução efetiva dos moldes impressos através de cada fatiador. As medições das alturas, comprimento e largura das peças impressas foram realizadas com um paquímetro digital (MIP/E-101, exatidão $\pm 0,02 \mathrm{~mm}$, Mitutoyo). Para as espessuras de $50 \mu \mathrm{m}$ e $100 \mu \mathrm{m}$ foram realizadas triplicatas das peças para o cálculo do valor médio de profundidade e a estimativa de erro associado, utilizando a ferramenta Excel® 2016 para o cálculo do desvio padrão.

Em seguida, foi realizada a análise de impressão utilizando as variáveis de microcanais. Por meio do processo de impressão 3D para fabricar dispositivos de caracterização, com base no fatiador com os melhores desempenhos dos testes anteriores, foram estudadas as variáveis presentes em um microcanal com micromisturador: largura do canal (W - 0,2 a $3 \mathrm{~mm}$ ), diâmetro dos obstáculos circulares ( $\mathrm{D}_{1}$ - 0,2 a 2,4 mm), distância entre o obstáculo e a parede do canal $\left(\mathrm{D}_{2}-0,3\right.$ a $\left.1 \mathrm{~mm}\right)$ e largura do obstáculo estático (E - 0,1 
a $0,4 \mathrm{~mm}$ ). Os dispositivos foram impressos para testar a resolução efetiva dos moldes impressos e determinar quais os menores valores para cada variável e a partir de qual valor a resolução de impressão é satisfatória. Os valores das variáveis foram medidos com auxílio de um microscópio digital (Dino-Lite Edge AM4115ZT, AnMo Electronics Corporation, Taiwan) e comparados com os valores obtidos pelo método de Microscopia Eletrônica de Varredura (MEV), realizada pelo Laboratório de Caracterização de Biomassa, Recursos Analíticos e de Calibração (LRAC - FEQ/UNICAMP).

\section{RESULTADOS E DISCUSSÕES}

As medidas da profundidade dos canais impressos com o polímero PLA utilizando os programas Simplify3D®, CuraEngine ${ }^{\circledR}$ e Slic3r ${ }^{\circledR}$, são apresentados nas Tabelas 1, 2 e 3, respectivamente. Observa-se que as peças com camadas de $300 \mu \mathrm{m}$ apresentaram valores múltiplos de $300 \mu \mathrm{m}$ como esperado, o que fez com que os valores impressos fossem diferentes dos valores propostos. As peças impressas com alturas de camada de $200 \mu \mathrm{m}$ apresentaram valores discrepantes com os especificados digitalmente, sendo, em sua maioria, menores que o proposto. Isto pode advir de uma má calibração da impressora para tal camada, uma vez que nos 3 programas houve valores muito longe do esperado. Em relação ao acabamento das peças, verificou-se que o Slic3r ${ }^{\circledR}$ apresenta um acabamento das peças inferior aos outros 2 programas, com aglomeração de material nas quinas das peças sendo mais notório conforme diminuí a espessura da camada de impressão. Pelo fato das peças com espessura de camada de $50 \mu \mathrm{m}$ e $100 \mu \mathrm{m}$ apresentarem melhores desempenhos, fez-se ensaios em triplicata para estes casos com os 3 programas.

Tabela 1 - Medidas da profundidade dos canais impressos com o polímero PLA utilizando o programa Simplify3D®, medido com paquímetro digital.

\begin{tabular}{|c|c|c|c|c|}
\hline $\begin{array}{c}\text { Altura } \\
\text { especificada } \\
(\mathrm{mm})\end{array}$ & $\begin{array}{c}\text { Profundidade } \\
\text { do canal para } \\
\text { camada de 50 } \\
\mu \mathrm{m}(\mathrm{mm})\end{array}$ & $\begin{array}{c}\text { Profundidade } \\
\text { do canal para } \\
\text { camada de 100 } \\
\mu \mathrm{m}(\mathrm{mm})\end{array}$ & $\begin{array}{c}\text { Profundidade } \\
\text { do canal para } \\
\text { camada de 200 } \\
\mu \mathrm{m}(\mathrm{mm})\end{array}$ & $\begin{array}{c}\text { Profundidade } \\
\text { do canal para } \\
\text { camada de 300 } \\
\mu \mathrm{m}(\mathrm{mm})\end{array}$ \\
\hline 0,2 & 0,20 & 0,18 & 0,15 & 0,31 \\
\hline 0,4 & 0,39 & 0,39 & 0,31 & 0,49 \\
\hline 1 & 0,97 & 0,91 & 0,72 & 1,15 \\
\hline 2 & 1,91 & 1,95 & 1,93 & 2,07 \\
\hline
\end{tabular}

Tabela 2 - Medidas da profundidade dos canais impressos com o polímero PLA utilizando o programa CuraEngine ${ }^{\circledR}$, medido com paquímetro digital.

\begin{tabular}{|c|c|c|c|c|}
\hline $\begin{array}{c}\text { Altura } \\
\text { especificada } \\
(\mathrm{mm})\end{array}$ & $\begin{array}{c}\text { Profundidade } \\
\text { do canal para } \\
\text { camada de 50 } \\
\mu \mathrm{m}(\mathrm{mm})\end{array}$ & $\begin{array}{c}\text { Profundidade } \\
\text { do canal para } \\
\text { camada de 100 } \\
\mu \mathrm{m}(\mathrm{mm})\end{array}$ & $\begin{array}{c}\text { Profundidade } \\
\text { do canal para } \\
\text { camada de 200 } \\
\mu \mathrm{m}(\mathrm{mm})\end{array}$ & $\begin{array}{c}\text { Profundidade } \\
\text { do canal para } \\
\text { camada de 300 } \\
\mu \mathrm{m}(\mathrm{mm})\end{array}$ \\
\hline \hline 0,2 & 0,20 & 0,18 & 0,17 & 0,27 \\
\hline 0,4 & 0,38 & 0,39 & 0,37 & 0,57 \\
\hline 1 & 0,92 & 0,99 & 0,77 & 1,17 \\
\hline 2 & 1,93 & 1,98 & 1,99 & 2,07 \\
\hline
\end{tabular}


Tabela 3 - Medidas da profundidade dos canais impressos com o polímero PLA utilizando o programa Slic3r®, medido com paquímetro digital.

\begin{tabular}{|c|c|c|c|c|}
\hline $\begin{array}{c}\text { Altura } \\
\text { especificada } \\
(\mathrm{mm})\end{array}$ & $\begin{array}{c}\text { Profundidade } \\
\text { do canal para } \\
\text { camada de 50 } \\
\mu \mathrm{m}(\mathrm{mm})\end{array}$ & $\begin{array}{c}\text { Profundidade } \\
\text { do canal para } \\
\text { camada de 100 } \\
\mu \mathrm{m}(\mathrm{mm})\end{array}$ & $\begin{array}{c}\text { Profundidade } \\
\text { do canal para } \\
\text { camada de 200 } \\
\mu \mathrm{m}(\mathrm{mm})\end{array}$ & $\begin{array}{c}\text { Profundidade } \\
\text { do canal para } \\
\text { camada de 300 } \\
\mu \mathrm{m}(\mathrm{mm})\end{array}$ \\
\hline \hline 0,2 & 0,14 & 0,19 & 0,24 & 0,20 \\
\hline 0,4 & 0,38 & 0,39 & 0,36 & 0,54 \\
\hline 1 & 1,04 & 1,01 & 0,99 & 1,20 \\
\hline 2 & 1,87 & 1,98 & 1,93 & 2,00 \\
\hline
\end{tabular}

Os resultados para a medição da profundidade em triplicatas são apresentados na Tabela 4. Foi verificado para todos os casos que a espessura de camada de $100 \mu \mathrm{m}$ apresentou precisão e exatidão melhores que os encontrados para a camada de $50 \mu \mathrm{m}$. Dentre os 3 programas de processamento de modelos $3 \mathrm{D}$, o CuraEngine ${ }^{\circledR}$ com espessura de camada de $100 \mu \mathrm{m}$, apresentou os melhores resultados com uma maior precisão e exatidão em relação aos demais. Ainda que o Slic3r® obteve resultados muito satisfatórios, o fato de seu acabamento superficial ser muito inferior aos demais o impossibilita de ser utilizado para a produção de microcanais. Por fim, em relação ao tempo de impressão, o Simplify3D® obteve os menores valores, aproximadamente $40 \%$ menores que dos demais programas, sendo uma opção viável para peças com tempo de impressão elevado.

Tabela 4 - Medidas da profundidade (altura) dos canais impressos (mm) com o polímero PLA utilizando o programa Simplify3D®, CuraEngine ${ }^{\circledR}$ e Slic3r®.

\begin{tabular}{|c|c|c|c|c|c|c|}
\hline $\begin{array}{c}\text { Altura } \\
\text { especificada } \\
(\mathrm{mm})\end{array}$ & $\begin{array}{c}\text { Altura do } \\
\text { canal para } \\
\text { camada de } \\
50 \mu \mathrm{m}\end{array}$ & $\begin{array}{c}\text { Altura do } \\
\text { canal para } \\
\text { camada de } \\
100 \mu \mathrm{m}\end{array}$ & $\begin{array}{c}\text { Altura do } \\
\text { canal para } \\
\text { camada de } \\
50 \mu \mathrm{m}\end{array}$ & $\begin{array}{c}\text { Altura do } \\
\text { canal para } \\
\text { camada de } \\
100 \mu \mathrm{m}\end{array}$ & $\begin{array}{c}\text { Altura do } \\
\text { canal para } \\
\text { camada de } \\
50 \mu \mathrm{m}\end{array}$ & $\begin{array}{c}\text { Altura do } \\
\text { canal para } \\
\text { camada de } \\
100 \mu \mathrm{m}\end{array}$ \\
\hline & \multicolumn{2}{|c|}{ Simplify3D® } & \multicolumn{2}{|c|}{ CuraEngine ${ }^{\circledR}$} & \multicolumn{2}{|c|}{ Slic3r® } \\
\hline 0,2 & $\begin{array}{c}0,18 \\
( \pm 0,02)\end{array}$ & $\begin{array}{c}0,18 \\
( \pm 0,02)\end{array}$ & $\begin{array}{c}0,19 \\
( \pm 0,01)\end{array}$ & $\begin{array}{c}0,19 \\
( \pm 0,01)\end{array}$ & $\begin{array}{c}0,17 \\
( \pm 0,03)\end{array}$ & $\begin{array}{c}0,19 \\
( \pm 0,01)\end{array}$ \\
\hline 0,4 & $\begin{array}{c}0,37 \\
( \pm 0,02)\end{array}$ & $\begin{array}{c}0,39 \\
( \pm 0,01)\end{array}$ & $\begin{array}{c}0,38 \\
( \pm 0,01)\end{array}$ & $\begin{array}{c}0,39 \\
( \pm 0,02)\end{array}$ & $\begin{array}{c}0,38 \\
( \pm 0,02)\end{array}$ & $\begin{array}{c}0,38 \\
( \pm 0,01)\end{array}$ \\
\hline 1 & $\begin{array}{c}0,93 \\
( \pm 0,03)\end{array}$ & $\begin{array}{c}0,97 \\
( \pm 0,05)\end{array}$ & $\begin{array}{c}0,96 \\
( \pm 0,04)\end{array}$ & $\begin{array}{c}0,98 \\
( \pm 0,03)\end{array}$ & $\begin{array}{c}1,00 \\
( \pm 0,04)\end{array}$ & $\begin{array}{c}1,00 \\
( \pm 0,01)\end{array}$ \\
\hline 2 & $\begin{array}{c}1,92 \\
( \pm 0,02)\end{array}$ & $\begin{array}{c}1,97 \\
( \pm 0,02)\end{array}$ & $\begin{array}{c}1,96 \\
( \pm 0,04)\end{array}$ & $\begin{array}{c}1,97 \\
( \pm 0,01) \\
\end{array}$ & $\begin{array}{c}1,94 \\
( \pm 0,06) \\
\end{array}$ & $\begin{array}{c}2,01 \\
( \pm 0,04)\end{array}$ \\
\hline
\end{tabular}

Foram verificadas as variáveis presentes em um projeto de microcanal com micromisturador, ou seja, a largura do canal, diâmetro dos obstáculos circulares, distância entre o obstáculo e a parede do canal, distância entre o conjunto de obstáculos no canal e largura do obstáculo estático. Em relação à largura do canal (Tabela 6), verificou-se que os microcanais apresentaram valores menores que o projetado, sendo que para valores de $\mathrm{W}$ menores que $1000 \mu \mathrm{m}$ o erro percentual aumenta drasticamente. Verificou-se que para $\mathrm{W}=$ $200 \mu \mathrm{m}$ o canal impresso era fechado, sendo este o valor limitante encontrado para a variável W. 
Em seguida foi verificado a resolução de impressão de obstáculos circulares/micromisturadores de $1 \mathrm{~mm}$ de altura e de diâmetro variável $\left(\mathrm{D}_{1}\right.$ - Tabela 6$)$. Verifica-se que os valores de impressão são menores que o projetado, sendo que para menores valores de $\mathrm{D}_{1}$, esta diferença tende a aumentar. Verificou-se que para $\mathrm{D}_{1}$ menor que $500 \mu \mathrm{m}$ não houve impressão, sendo este o valor limitante encontrado para a variável $\mathrm{D}_{1}$ devido ao fato do bico extrusor apresentar diâmetro interno de 0,4 $\mathrm{mm}$. Em relação a distância entre os obstáculos circulares e as paredes do canal (Tabela 7), os diâmetros finais dos pilares impressos foram menores que os projetados, o que resultou em estruturas com múltiplos pilares sangrando uns nos outros (i.e., presença de material de um pilar na próxima estrutura) quando o valor de $\mathrm{D}_{2}$ não era suficientemente grande para compensar a propagação de gotículas. Este fator foi verificado no trabalho de Hwang et al. (2015). Verificou-se que conforme o valor dos diâmetros dos obstáculos aumenta, o valor $\mathrm{D}_{2}$ se afasta mais do projetado sendo verificados valores menores. Foi verificado que para $\mathrm{D}_{1}$ acima de $0,5 \mathrm{~mm}$, valores de $\mathrm{D}_{2}$ acima de $0,625 \mathrm{~mm}$ não promovem o sangramento entre os pilares ou tem o sangramento muito reduzido, sendo considerado o valor mínimo para o passo entre os obstáculos circulares.

Por fim, em relação a variável E, apenas ocorreu impressão para E igual a 0,4 mm sendo que o valor obtido na impressão foi de $0,8 \mathrm{~mm}$. Este valor mínimo é devido ao bico extrusor ser de 0,4 mm. Este valor mínimo para E está muito distante dos valores para serem utilizados como obstáculos de microcanais, impossibilitando, a primeiro momento, de projetar futuros microcanais com obstáculos estáticos por meio desta impressora.

Tabela 5 - Largura dos canais impressos (W) medidos através da MEV e com auxílio do Microscópio Digital.

\begin{tabular}{|c|c|c|}
\hline $\begin{array}{c}\text { W } \\
\text { especificado } \\
(\mu \mathrm{m})\end{array}$ & $\begin{array}{c}\text { W impresso medido } \\
\text { através da MEV } \\
(\mu \mathrm{m})\end{array}$ & $\begin{array}{c}\text { W impresso medido } \\
\text { através do Microscópio } \\
\text { Digital }(\mu \mathrm{m})\end{array}$ \\
\hline \hline 500 & 340,13 & 350 \\
\hline 1000 & 892,35 & 850 \\
\hline 1500 & 1280 & 1320 \\
\hline 3000 & 2610 & 2780 \\
\hline
\end{tabular}

Tabela 6 - Largura dos diâmetros dos obstáculos circulares impressos $\left(\mathrm{D}_{1}\right)$ medidos através da MEV e com auxílio do Microscópio Digital.

\begin{tabular}{|c|c|c|}
\hline $\begin{array}{c}\mathrm{D}_{1} \\
\text { especificado } \\
(\mathrm{mm})\end{array}$ & $\begin{array}{c}\mathrm{D}_{1} \text { impresso medido } \\
\text { através da MEV } \\
(\mathrm{mm})\end{array}$ & $\begin{array}{c}\mathrm{D}_{1} \text { impresso medido } \\
\text { através do Microscópio } \\
\text { Digital }(\mathrm{mm})\end{array}$ \\
\hline \hline 0,50 & 0,473 & -- \\
\hline 0,75 & 0,882 & 0,780 \\
\hline 1,00 & 1,15 & 1,000 \\
\hline 1,50 & 1,56 & 1,500 \\
\hline 2,00 & 1,98 & 2,000 \\
\hline 2,30 & 2,27 & 2,300 \\
\hline 2,40 & 2,35 & 2,400 \\
\hline 2,48 & 2,35 & 2,480 \\
\hline
\end{tabular}


Tabela 7 - Largura das distâncias entre os obstáculos circulares e as paredes dos canais impressos $\left(\mathrm{D}_{2}\right)$ medidos através da MEV e do Microscópio Digital para $\mathrm{D}_{1}=1000$ e $1500 \mu \mathrm{m}$.

\begin{tabular}{|c|c|c|c|c|c|}
\hline $\begin{array}{c}\mathrm{D}_{2} \\
\text { especificado } \\
(\mu \mathrm{m})\end{array}$ & $\begin{array}{c}\mathrm{D}_{2} \text { impresso } \\
\text { medido } \\
\text { através da } \\
\text { MEV }(\mu \mathrm{m})\end{array}$ & $\begin{array}{c}\mathrm{D}_{2} \text { impresso } \\
\text { medido através } \\
\text { do } \\
\text { Microscópio } \\
\text { Digital }(\mu \mathrm{m})\end{array}$ & $\begin{array}{c}\mathrm{D}_{2} \\
\text { especificado } \\
(\mu \mathrm{m})\end{array}$ & $\begin{array}{c}\mathrm{D}_{2} \text { impresso } \\
\text { medido } \\
\text { através da } \\
\mathrm{MEV}(\mu \mathrm{m})\end{array}$ & $\begin{array}{c}\mathrm{D}_{2} \text { impresso } \\
\text { medido através } \\
\text { do } \\
\text { Microscópio } \\
\text { Digital }(\mu \mathrm{m})\end{array}$ \\
\hline \hline \multicolumn{3}{|c|}{$\mathrm{D}_{1}=1000 \mu \mathrm{m}$} & \multicolumn{3}{|c|}{$\mathrm{D}_{1}=1500 \mu \mathrm{m}$} \\
\hline \hline 300 & 346,04 & 300 & 500 & 361,39 & 400 \\
\hline 500 & 497,77 & 500 & 750 & 542,02 & 600 \\
\hline 625 & 652,51 & 900 & 1000 & 693,98 & 1000 \\
\hline 1000 & 1030 & 1000 & & & \\
\hline
\end{tabular}

\section{CONCLUSÃO}

A produção de microcanais por meio de impressão 3D foi descrita, estudando as variáveis que mais influenciam na fabricação dos mesmos. A utilização do programa CuraEngine ${ }^{\circledR}$ imprimindo PLA à espessura de camada de $100 \mu \mathrm{m}$ obteve os melhores resultados de resolução de impressão. As variáveis presentes nos microcanais foram estudadas para a impressora Sethi3D S3 utilizando o programa de processamento de modelos 3D CuraEngine ${ }^{\circledR}$ e imprimindo com PLA, obtendo os valores mínimos de cada variável, verificando que é viável a fabricação de microcanais por este método.

\section{REFERENCIAS}

COLTRO, W. K. T.; PICCIN, E.; CARRILHO, E.; JESUS, D. P. de.; SILVA, A. F. da.; SILVA, H. D. T. da.; LAGO, C. L. Microssistemas de análises químicas. Introdução, tecnologias de fabricação, instrumentação e aplicações. Química Nova, v. 30, p. 19862000, 2007.

DAI, J.Y.; LI, D.Y.; ZHAO, Y. C.; XIU, Z. L. Statistical optimization for biodiesel production from soybean oil in a microchannel reactor. Industrial \& Engineering Chemistry Research, v. 53, p. 9325-9330, 2014.

HWANG, YONGHA; PAYDAR, OMEED H.; CANDLER, ROBERT N. 3D printed molds for non-planar PDMS microfluidic channels. Sensors and Actuators A: Physical, v. 226, p. 137-142, 2015.

KITSON, P. J.; GLATZEL, S.; CHEN, W.; LIN, C. G.; SONG, Y. F.; CRONIN, L. 3D printing of versatile reactionware for chemical synthesis. Nature protocols, v 11:5, 920936, 2016.

LEE, J. M.; ZHANG, M.; YEONG, W. Y. Characterization and evaluation of 3D printed microfluidic chip for cell processing. Microfluid and Nanofluid, v. 20:5, 2016. 